Check for updates

Cite this: RSC Adv., 2018, 8, 12708

Received 30th January 2018

Accepted 14th March 2018

DOI: $10.1039 / c 8 \mathrm{ra00917a}$

rsc.li/rsc-advances

\section{Semi-quantitative and visual assay of copper ions by fluorescent test paper constructed with dual- emission carbon dots $\uparrow$}

\begin{abstract}
Yifan Wang, ${ }^{a}$ Mian $\mathrm{Wu}^{\mathrm{a}}$ Shaoming $\mathrm{Yu}^{\star \mathrm{a}}$ and Changlong Jiang (iD) *bc
A novel, simple and effective dual-emissive fluorescent probe for the sensitive and selective detection of $\mathrm{Cu}(I)$ has been developed by mixing blue carbon dots and orange carbon dots, with a sensitive detection limit of $7.31 \mathrm{nM}$. The blue fluorescence can be selectively quenched by $\mathrm{Cu}\left({ }^{\prime \prime}\right)$, while the orange fluorescence is a internal reference, resulting in a distinguishable fluorescence color change from blue to orange under a UV lamp. Meanwhile, its as-prepared text paper provides a convenient and simple approach for the visual detection of $\mathrm{Cu}(॥)$ and successfully applied in real water samples, with a dosediscerning ability as low as $50 \mathrm{nM}$. The methodology reported here opens a novel pathway toward the real applications of fluorescent test papers.
\end{abstract}

\section{Introduction}

In addition to zinc and iron, copper is the third trace metal element that is necessary for human health. Copper ions $(\mathrm{Cu}(\mathrm{II}))$, as heavy metal ions and essential components of many enzyme systems, play an important role in some physiological and pathological processes, such as serving as a significant catalytic cofactor for the synthesis of collagen, elastin and hemoglobin. ${ }^{1-3}$ An excess $\mathrm{Cu}(\mathrm{II})$ concentration may become toxic to living organisms and induce damage to the kidneys, liver and the central nervous system. And the widespread use of $\mathrm{Cu}(\mathrm{II})$ in industry and agriculture can also lead to serious copper pollution in water and soil. ${ }^{4,5}$ Meanwhile, the deficiency of $\mathrm{Cu}$ (II) can lead to many diseases such as bone abnormalities and anemia. ${ }^{6}$ The maximum allowable level of $\mathrm{Cu}$ (II) in drinking water, defined by United States Environmental Protection Agency (EPA), is $20 \mu \mathrm{M}^{7}$ Therefore, it is very important to establish an efficient method for sensitive determination of trace copper, with regard to the associated health concerns and environmental monitoring. Although conventional analytical techniques including voltammetry and potentiometry, ${ }^{8}$ inductively coupled plasma mass spectroscopy, ${ }^{9}$ atomic absorption spectroscopy/emission spectroscopy, ${ }^{10}$ could meet the demand of sensitive and selective measurement of $\mathrm{Cu}(\mathrm{II})$ in water

${ }^{a}$ School of Chemistry and Chemical Engineering, Hefei University of Technology, Hefei, Anhui, 230009, China. E-mail: shmyu@hfut.edu.cn

${ }^{b}$ Institute of Intelligent Machines, Chinese Academy of Sciences, Hefei, Anhui, 230031, China.E-mail: cljiang@iim.ac.cn

'State Key Laboratory of Transducer Technology, Chinese Academy of Sciences, Hefei, Anhui, 230031, China. E-mail: cljiang@iim.ac.cn

$\dagger$ Electronic supplementary information (ESI) available. See DOI: 10.1039/c8ra00917a samples, all these methods are not only time-consuming, laborintensive, and laboratory-based but also require expensive instrumentation and large sample volume. In this frame, there is a crucial requirement for the development of reliable, fast, and cheap techniques to detect $\mathrm{Cu}$ (II) in water samples.

Recently, various fluorescent sensors including organic dyes, quantum dots (QDs) and carbon dots (CDs), with the festures of simplicity, high sensitivity, good selectivity and rapid response, show great potential in the sensitive detection of $\mathrm{Cu}$ (II). Moreover, fluorescent sensors possess another unparallel advantage, that is, their visualization capability for the determination of analyte with the naked eye by the aid of a simple ultraviolet (UV) lamp. Owing to the classical success of $\mathrm{pH}$ test paper, the fluorescent test papers have been widely explored by assembling. or printing the fluorescent probes onto a piece of paper-based substrates for the visual assays with low cost, easy operation, and portable feasibility, for example, our group has innovated the color-multiplexing fluorescent test papers by the cooperative employments of QDs and CDs for the visual detections of arsenic ions ${ }^{11}$ and blood sugar, ${ }^{12}$ in which one of two fluorophores were used as the internal color standard to enhance the visualization contrast. The obtained dosage-sensitive color evolution was similar to the performance of $\mathrm{pH}$ test paper, but the bad compatibility between QDs and CDs renders the preparation procedure very tedious and the environmental toxicity of QDs also limit the usage of test papers. As is known to all, organic dyes often suffer from fast-photobleaching, low fluorescence quantum yield, narrow excitation spectra. ${ }^{13,14}$ And most QDs are based on semiconductors that contain heavy metals, such as cadmium, and their applications are thus limited for well-known toxicity and high cost. ${ }^{15-17}$ In comparison with the organic dyes and QDs, CDs possess many outstanding advantages, such as low toxicity, biocompatibility, low cost and 
chemical inertness in addition to having similar fluorescence properties. ${ }^{18}$

Herein, we report a novel dual-emissive fluorescent probe and its as-prepared text paper for the visual detection of $\mathrm{Cu}(\mathrm{II})$. The dual-emission ratiometric fluorescence probe that prepared by mixing blue CDs (BCDs) and orange CDs (OCDs) with fluorescence intensity ratio of $4: 1$ possesses two emission peaks at $440 \mathrm{~nm}$ and $610 \mathrm{~nm}$ under a single wavelength excitation of $360 \mathrm{~nm}$. The blue fluorescence of the BCDs can be quenched by $\mathrm{Cu}$ (II), while the orange fluorescence of the OCDs is insensitive to the analyte. The control of emissive intensity on the blue and orange fluorescences in the probe allows the color evolution from blue to orange with the concentrations of $\mathrm{Cu}$ (II), which can be conveniently observed by the naked eye under a UV lamp without any complicated instrumentation.

\section{Experimental section}

\subsection{Reagents and instruments}

Citric acid, polyethylenimine $(M=600)$, ethanol and all metal salts were supplied by Sinopharm Chemical Reagent Company, Ltd. (Shanghai, China). P-phenylenediamine ( $p$-PDA) was purchased from Sigma-Aldrich. All chemicals were used as received without further purification unless otherwise specified. Ultrapure water $(18.2 \mathrm{M} \Omega \mathrm{cm})$ was produced with a Millipore water purification system.

Fluorescence measurement was recorded on a Perkin-Elmer LS-55 luminescence spectrometer (Liantriant, UK). The structures and morphologies of CDs were examined using a JEOL 2010 transmission electron microscope. The UV-visible absorption spectra were obtained with a Shimadzu UV-2550 spectrometer. Infrared spectra of infrared spectra of the dried CDs etc. dispersed in $\mathrm{KBr}$ pellets were recorded on a ThermoFisher Nicolet iS10 FT-IR spectrometer. Fluorescent photos were taken under AGL-9406 portable UV lamp $(254 \mathrm{~nm})$ by a Canon 350 D digital camera.

\subsection{Synthesis of BCDs}

BCDs were synthesized through a simple Hydrothermal method. ${ }^{19} 1.0 \mathrm{~g}$ citric acid dissolved in $30 \mathrm{~mL}$ ultrapure water firstly. Subsequently, $0.5 \mathrm{~g}$ polyethylenimine was added to the solution. Finally, the mixed solution was transferred to a poly(tetrafluoroethylene)-lined autoclave, and heated at $200{ }^{\circ} \mathrm{C}$ for $6 \mathrm{~h}$. After cooling to room temperature naturally the resultant BCDs were purified by dialysis for $72 \mathrm{~h}$. The purified BCDs were dispersed in ultrapure water $\left(0.03 \mathrm{mg} \mathrm{mL}^{-1}\right)$ and stored at $4{ }^{\circ} \mathrm{C}$ for further use.

\subsection{Synthesis of OCDs}

OCDs were prepared according to the reported solvothermal method with a minor modification. ${ }^{20} 0.3 \mathrm{~g}$ of $p$-PDA was dissolved in $30 \mathrm{~mL}$ ethanol, and the solution was subsequently transferred into a poly(tetrafluoroethylene)-lined autoclave. After heated at $180{ }^{\circ} \mathrm{C}$ for $12 \mathrm{~h}$ and then cooled down to room temperature naturally, the mixture was purified through a silica chromatography column using ethyl acetate as eluent. The final product was obtained by drying in a rotary evaporator, and the purified OCDs were redispersed in ultrapure water $(0.07 \mathrm{mg}$ $\mathrm{mL}^{-1}$ ) and stored at $4{ }^{\circ} \mathrm{C}$ for further use.

\subsection{Preparation of test papers}

Washed the commercial ink cartridge completely with ultrapure water until the ink powder was cleared away, and dried it in an oven at $60{ }^{\circ} \mathrm{C}$ for $4 \mathrm{~h}$. Then, the ink replaced by the ratiometric fluorescence probe was injected into the vacant cartridge, and a filter paper was sticked onto a piece of A4 paper. The ratiometric fluorescence probe was printed on the filter paper through an inkjet printer connected with a computer, and the printing process was repeated $10-20$ times to enhance the amount of probe. Finally, the filter paper displayed strong blue fluorescent under a $365 \mathrm{~nm}$ UV lamp.

\subsection{Detections of $\mathrm{Cu}(\mathrm{II})$}

$\mathrm{Cu}$ (II) with different concentrations were added to the ratiometric fluorescent probe solution which is prepared by mixing BCDs and OCDs with the fluorescence intensity ratio of $4: 1$ in $2 \mathrm{~mL}$ of HEPES buffer $(\mathrm{pH}=7.4)$, and reacted for $3 \mathrm{~min}$. The resulting fluorescence spectra were recorded by a fluorescence spectrometer.

Detections of $\mathrm{Cu}$ (II) on test paper: different concentrations of $\mathrm{Cu}(\mathrm{II})$ dropped onto the as-prepared test paper, and subsequently the color changes of test paper were observed under a $365 \mathrm{~nm}$ UV lamp.

\subsection{Analysis of $\mathrm{Cu}(\mathrm{II})$ in tap water and lake water samples}

Detections of $\mathrm{Cu}$ (II) in real water samples including tap water and lake water was performed to evaluate the applicability of the fluorescent probe in environmental samples. Tap water were obtained from our lab and the lake water sample was collected from a local lake. The water samples collected were filtered by ordinary qualitative filter paper and $0.45 \mu \mathrm{m}$ Supor filters to remove the impurities. Then, different concentrations of $\mathrm{Cu}$ (II) were added into the as-prepared water samples and these samples were analyzed by the fluorescent probes. The average was obtained from three independent measurements and presented with a standard deviation. And the samples were also analyzed by the test paper.

\section{Results and discussion}

The BCDs' properties were characterized by transmission electron microscopy (TEM), UV-vis absorption, FTIR spectroscopy, and fluorescence spectroscopy. As shown in Fig. 1A, the asprepared BCDs have a good monodispersity with a diameter of $\sim 5 \mathrm{~nm}$. And, the BCDs present two characteristic absorption at $248 \mathrm{~nm}$ and $354 \mathrm{~nm}$, which are ascribed to the large amount of $\pi$-electrons in the $\mathrm{sp}^{2}$-hybridized structure and the $\mathrm{n}-\pi^{*}$ transition of CDs, respectively (the red curve in Fig. S1A $\dagger$ ). ${ }^{21,22}$ The fluorescence spectrum of BCDs exhibited an emission peak at $440 \mathrm{~nm}$ with an excitation of $360 \mathrm{~nm}$ (the black curve in Fig. S1A $\dagger$ ), and a bright blue fluorescence was under a $365 \mathrm{~nm}$ UV lamp (inset in Fig. S1A $\dagger$ ). Moreover, the FT-IR spectrum was 


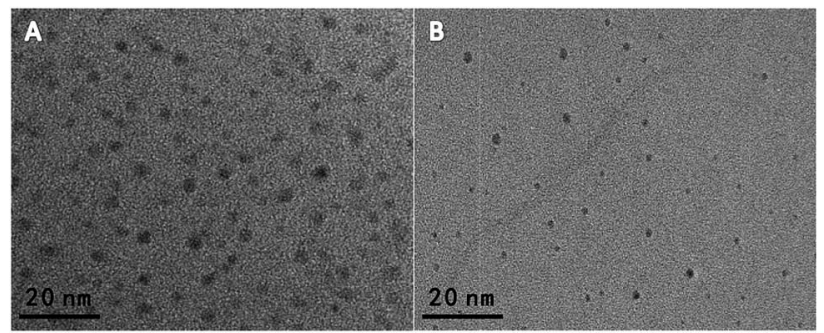

Fig. 1 TEM image (A) of BCDs and (B) of OCDs.

carried out to obtain further structural insights about the BCDs. As shown in Fig. S2A, $\uparrow$ the absorption bands at 1376, 1441 and $2944 \mathrm{~cm}^{-1}$ are attributed to $\mathrm{C}-\mathrm{H}$ bending vibrations. Additionally, the peak at $1648 \mathrm{~cm}^{-1}$, the strong peak of $1562 \mathrm{~cm}^{-1}$, and $1300 \mathrm{~cm}^{-1}$ are attributed to amide $\mathrm{C}=\mathrm{O}, \mathrm{N}-\mathrm{H}$, and $\mathrm{C}-\mathrm{N}$ respectively. ${ }^{23}$ And the absorption band at $3420 \mathrm{~cm}^{-1}$ suggests the presence of $-\mathrm{NH}$ and/or - $\mathrm{OH}$ groups, which is propitious to improve the hydrophilicity of BCDs solution. ${ }^{19}$ As shown in Fig. 1B, the obtained $\sim 3 \mathrm{~nm}$ sized OCDs were highly monodispersive. And, the OCDs exhibited a orange emission centered at $610 \mathrm{~nm}$ with the excitation of $360 \mathrm{~nm}$ (Fig. S1B $\dagger$ ), and a bright orange fluorescence was observed under a $365 \mathrm{~nm}$ UV lamp (the inset image of Fig. S1B $\dagger$ ). The UV-vis spectrum has two characteristic absorption peaks centered at $239 \mathrm{~nm}$ and $281 \mathrm{~nm}$ (the red line in Fig. $\mathrm{S} 1 \mathrm{~B} \dagger$ ), and the two peaks are assigned to the $\pi-$ $\pi^{*}$ transitions of $\mathrm{C}=\mathrm{C}$ and $\mathrm{C}=\mathrm{N}$ bonds in the aromatic rings, respectively, which are identical to those of $p$-PDA. ${ }^{24-26}$ Additionally, in FT-IR spectrum of Fig. S2B, $\uparrow$ the two sharp peaks at 1635 and $1515 \mathrm{~cm}^{-1}$ ascribed to the $\mathrm{C}=\mathrm{N}$ and $\mathrm{C}=\mathrm{C}$ stretching vibrations, respectively, and the peak at $818 \mathrm{~cm}^{-1}$ corresponded to the out-plane bending of benzene ring. ${ }^{20,24,25}$
As shown in Fig. S3, $\uparrow$ the fluorescence excitation peaks of the BCDs are at $218 \mathrm{~nm}, 245 \mathrm{~nm}$ and $354 \mathrm{~nm}$, and those of the OCDs are at 280, 365 and $484 \mathrm{~nm}$. Moreover, as illustrated in Fig. S4, $\dagger$ the fluorescence intensities of the BCDs shows a tendency to rise first and then decline by adjusting the excitation wavelength from $330 \mathrm{~nm}$ to $370 \mathrm{~nm}$. Similarly, the fluorescence intensities of the OCDs shows a same tendency by adjusting the excitation wavelength from $340 \mathrm{~nm}$ to $380 \mathrm{~nm}$. So, in order to excite the BCDs and OCDs simultaneously, we choose $360 \mathrm{~nm}$ as the excitation band for the ratiometric fluorescence probe. As shown in the fluorescence spectra of the BCDs, OCDs and ratiometric probe (Fig. S5 $\dagger$ ), the BCDs and OCDs show a maximum emission at 440 and $610 \mathrm{~nm}$, respectively, and the ratiometric probe which disperses well in water can exhibit dual-emission bands at $440 \mathrm{~nm}$ and $610 \mathrm{~nm}$ under a single wavelength excitation. As shown in Fig. S6, $\uparrow$ the stability of the ratiometric probe is systematically investigated by fluorescence spectra in aqueous solution. The fluorescence intensity ratios $\left(I_{440} / I_{610}\right)$ of the probe remain unchanged over $2 \mathrm{~h}$, demonstrating its excellent photostability.

Fig. S7† presents the emission spectra of the OCDs in response to $\mathrm{Cu}$ (II), showing that the fluorescence spectra remain constant without an obvious change of fluorescence color upon the addition of $\mathrm{Cu}(\mathrm{II})$ up to a concentration of $500 \mathrm{nM}$. Conversely, the fluorescence spectra of the BCDs can be greatly quenched upon gradual addition of $\mathrm{Cu}$ (II) from 0 to $500 \mathrm{nM}$, but the color change of the single fluorescence quenching of the BCDs is hard to be distinguished by the naked eyes compared with the ratiometric probe. The structure of the dual-emission fluorescent probe and the working principle for visual detection of $\mathrm{Cu}(\mathrm{II})$ are illustrated in Scheme 1. To design the ratiometric fluorescence probe, the BCDs act as a reaction site for $\mathrm{Cu}(\mathrm{II})$, in which the blue fluorescence is effectively quenched by

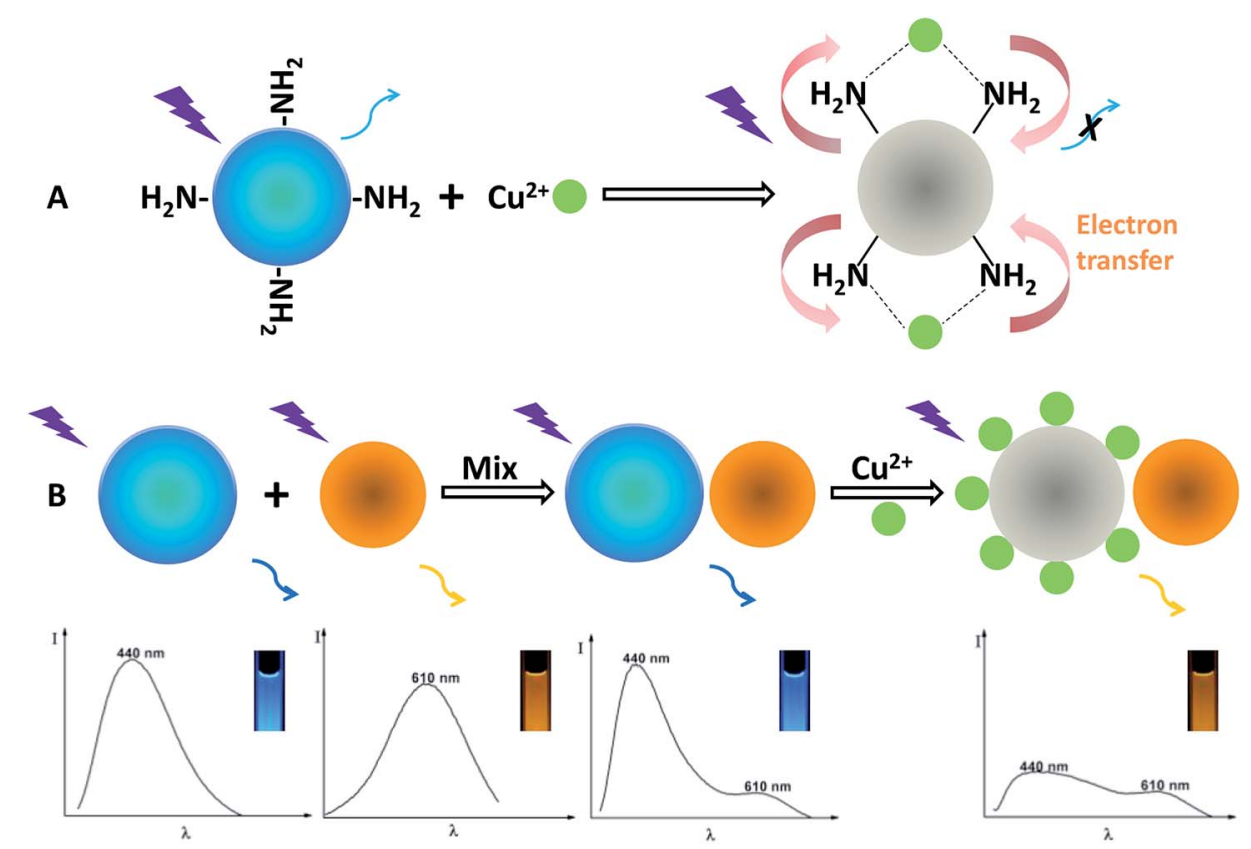

Scheme 1 Schematic illustration of the visual detection principle for $\mathrm{Cu}(॥)$ and the formation of the dual-emission ratiometric fluorescence probe. 
$\mathrm{Cu}(\mathrm{II})$ (Fig. S7A $\dagger$ ), and OCDs are selected as the reference signal in the fluorescent probe due to its good photostability and chemical inertness in the presence of $\mathrm{Cu}$ (II) (Fig. S7B $\dagger$ ). When $\mathrm{Cu}$ (II) is present, the complexation between $\mathrm{Cu}$ (II) and amino groups of BCDs leads to the splitting of $d$ orbital of $\mathrm{Cu}(\mathrm{II})$. Therefore, electrons in the excited state of BCDs have an opportunity to transfer to the d orbital of $\mathrm{Cu}$ (II). Electron transition in the radiation form (fluorescence emission) of BCDs is consequently restrained, leading to fluorescence quenching. ${ }^{19}$

We have investigated three different ratio of blue/orange to obtain the widest color variation with the mixture of blue and orange. BCDs and OCDs are mixed and the ratio of emission intensity of blue to orange was adjusted to $4: 1$ (Fig. S8 $\dagger$ ), which could display a very wide/consecutive luminescence "from blue to orange". As shown in Fig. 2A, for evaluating the sensitivity of the ratiometric probe, fluorescence responses were measured upon the addition of different amounts of $\mathrm{Cu}(\mathrm{II})$. The slight variation of the intensity ratios of the two emission peaks lead to a distinguishable fluorescence color change from blue to purple, to pink and to orange, which is available for the visual detection of $\mathrm{Cu}(\mathrm{II})$ by the naked eye. Fig. 2B shows that the fluorescence intensity ratio $\left(I_{440} / I_{610}\right)$ is closely related to the concentration of $\mathrm{Cu}(\mathrm{II})$. To quantitatively evaluate the amounts of $\mathrm{Cu}$ (II), a good linear relationship $\left(R^{2}=0.9968\right)$ for the concentration of $\mathrm{Cu}(\mathrm{II})$ ranging from 0 to $500 \mathrm{nM}$ was obtained
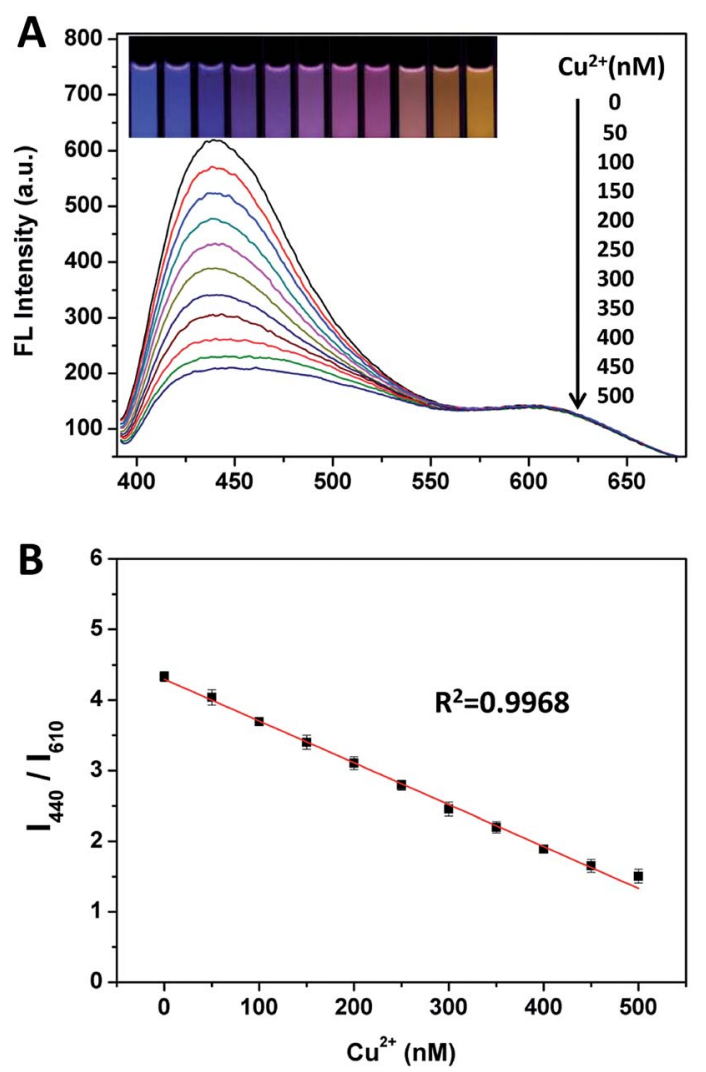

Fig. 2 (A) The fluorescent spectra of mixture of BCDs and OCDs at ratios $4: 1$ with the addition of $\mathrm{Cu}\left({ }_{11}\right)$. The inset shows the corresponding fluorescent photo under $365 \mathrm{~nm}$ UV lamp. (B) The plot of $I_{440} / I_{610}$ of the ratiometric probe versus $\mathrm{CU}(\mathrm{II})$ concentrations. by plotting the $I_{440} / I_{610}$ ratio versus the concentrations of $\mathrm{Cu}$ (II). The detection limit, which was defined as 3 times the standard deviation of background $(3 \sigma)$, was calculated to be as low as $7.31 \mathrm{nM}$. The dynamic experiment demonstrated that the fluorescence response to $\mathrm{Cu}^{2+}$ was completed in $\sim 3 \mathrm{~min}$ (Fig. $\mathrm{S} 9 \dagger$ ).

The fluorescent intensity ratio $\left(I_{380} / I_{620}\right)$ was measured by adding various metal ions in the ratiometric fluorescent probe solution at same conditions to examine the selectivity of the ratiometric fluorescence probe for $\mathrm{Cu}(\mathrm{II})$. As shown in Fig. 3 and $\mathrm{S} 10, \uparrow$ while the $I_{440} / I_{610}$ ratio is quenched about $80 \%$ by $\mathrm{Cu}(\mathrm{II})$ at $0.5 \mu \mathrm{M}$, no obvious change in $I_{440} / I_{610}$ and fluorescent color was detected with the additions of $5 \mu \mathrm{M} \mathrm{Na}{ }^{+}, \mathrm{K}^{+}, \mathrm{Zn}^{2+}, \mathrm{Al}^{3+}, \mathrm{Ni}^{2+}$, $\mathrm{As}^{3+}, \mathrm{Li}^{+}, \mathrm{Fe}^{3+}, \mathrm{Ag}^{+}, \mathrm{Co}^{2+}, \mathrm{Hg}^{2+}, \mathrm{Mn}^{2+}, \mathrm{Ba}^{2+}, \mathrm{Ca}^{2+}, \mathrm{Cd}^{2+}, \mathrm{Mg}^{2+}$, and $\mathrm{Pb}^{2+}$ into the probe solution. Moreover, the simultaneous addition of $5 \mu \mathrm{M} \mathrm{Na}{ }^{+}, \mathrm{K}^{+}, \mathrm{Zn}^{2+}, \mathrm{Al}^{3+}, \mathrm{Ni}^{2+}, \mathrm{As}^{3+}, \mathrm{Li}^{+}, \mathrm{Fe}^{3+}, \mathrm{Ag}^{+}$, $\mathrm{Co}^{2+}, \mathrm{Mn}^{2+}, \mathrm{Ba}^{2+}, \mathrm{Ca}^{2+}, \mathrm{Cd}^{2+}, \mathrm{Mg}^{2+}$, and $\mathrm{Pb}^{2+}$ into the probe solution quenched the $I_{440} / I_{610}$ ratio by only about $20 \%$ (Fig. S11 $\dagger$ ). However, after further addition of $500 \mathrm{nM} \mathrm{Cu}^{2+}$, the fluorescence intensity ratio $\left(I_{440} / I_{610}\right)$ of the probe changes greatly. It should be noted here that, in the complexation reactions with amines, $\mathrm{Ag}^{+}$and $\mathrm{Cu}^{2+}$ may have similar reactivities. However, $\mathrm{Ag}^{+}$does not inhibit the fluorescence response of $\mathrm{CD}$, and the presence of $\mathrm{Ag}^{+}$does not affect the quenching activity of $\mathrm{Cu}^{2+}$ either, this might result from the formed of silver amine complexes. ${ }^{27}$ It is noted that $\mathrm{Hg}^{2+}$ can slightly quench the fluorescence of the probe, but its interference can easily be suppressed by a simple sample pretreatment with KI, $\mathrm{NaCl}$, and rhodamine $\mathrm{B}^{28}$ These data indicate that the ratiometric fluorescent probe exhibits excellent selectivity for $\mathrm{Cu}(\mathrm{II})$.

The feasibility of the ratiometric fluorescent probe for detecting $\mathrm{Cu}$ (II) in real samples was explored by natural water (lake water and tap water). Tap water and lake water were first filtered to remove the undissolved substances, and then spiked with $\mathrm{Cu}$ (II) at the concentrations of 200,300 and $400 \mathrm{nM}$. The results are summarized in Table 1 . For the three samples, they both have similar results and good recovery rates range from

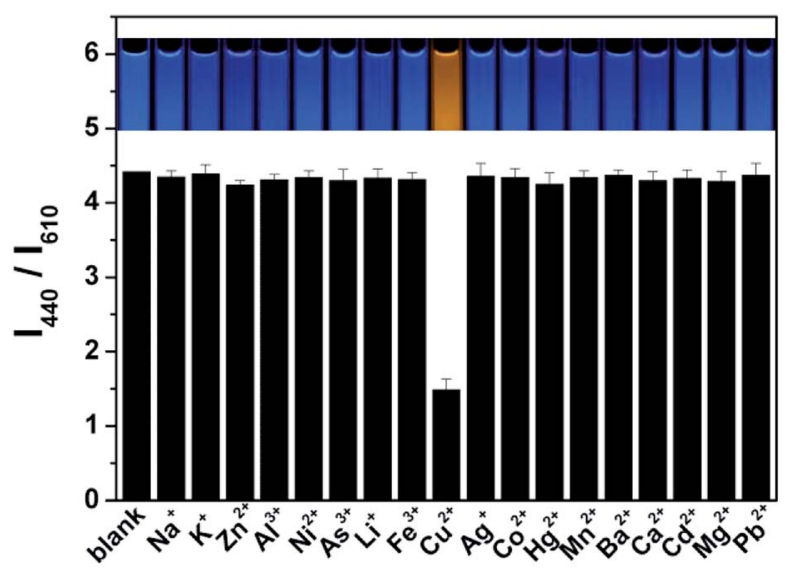

Fig. 3 The ratiometric fluorescent responses to various metallic ions and. The selectivity tests were done in HEPES buffer $(\mathrm{pH}=7.4)$ with the addition of $5 \mu \mathrm{M}$ various metallic ions and $0.5 \mu \mathrm{M} \mathrm{Cu}(॥)$ into the mixing BCDs/OCDs ( $4: 1$ in fluorescent intensity). The inset images show the corresponding fluorescent photos under a $365 \mathrm{~nm}$ UV lamp. 
Table 1 The recoveries of $\mathrm{Cu}(\mathrm{II})$ in tap water and lake water by the measurements of ratiometric fluorescence

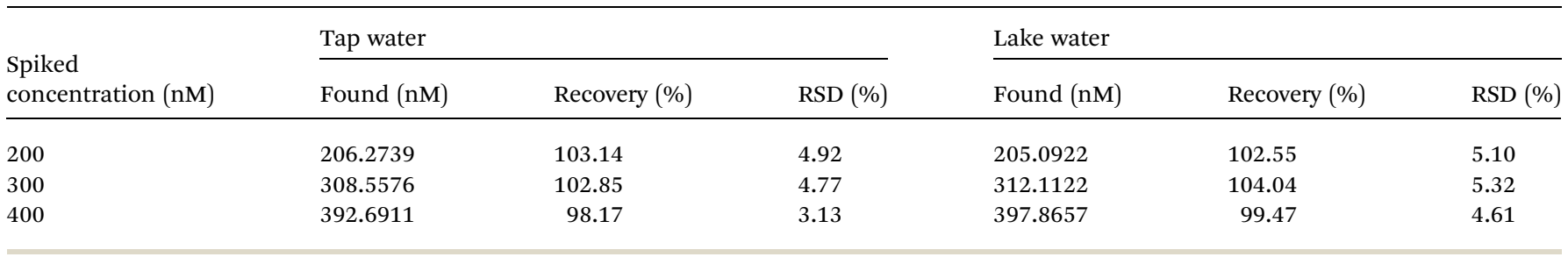

98.17-104.04\%. These results imply that the ratiometric fluorescent probe is likely to be capable of practically useful $\mathrm{Cu}$ (II) detection upon further development.

Following the above strategy, we have prepared fluorescent test paper for the visual semiquantification detection of $\mathrm{Cu}$ (II). The BCDs and OCDs were mixed and adjusted to a ratiometric fluorescent intensity of $4: 1$, and the aqueous mixture was used as fluorescent ink. The test papers with the fluorescent ink displayed a highly uniform blue brightness on the whole piece of paper under the irradiation of UV lamp at $365 \mathrm{~nm}$. As shown in Fig. 4A, while dropping the aqueous solution of $\mathrm{Cu}$ (II) onto the test paper, the colors of test papers under UV lamp consecutively and gradually evolved from blue to purple to pink and to orange with the increment of $\mathrm{Cu}$ (II) concentration from 0 to 500 nM. Fig. S12† also shows that the temporal color evolution with the addition of $500 \mathrm{nM} \mathrm{Cu(II)} \mathrm{displayed} \mathrm{a} \mathrm{serial} \mathrm{of}$ intermediate colors from blue to orange in $4 \mathrm{~min}$. We further examined the applicability of the fluorescent test papers for the detection of $\mathrm{Cu}(\mathrm{II})$ in real water samples. The fluorescent test papers gave out the obvious color responses to the different $\mathrm{Cu}(\mathrm{II})$ concentrations, and the corresponding colors are identical in the cases of tap water and lake water (Fig. 4B and C). Moreover, the tendency of color evolutions for the detection of real samples is very similar to that in Fig. 4A. The excellent visual effect and accuracy suggest that the fluorescent test papers can meet with the requirements for the visual detection of $\mathrm{Cu}(\mathrm{II})$ in water samples.
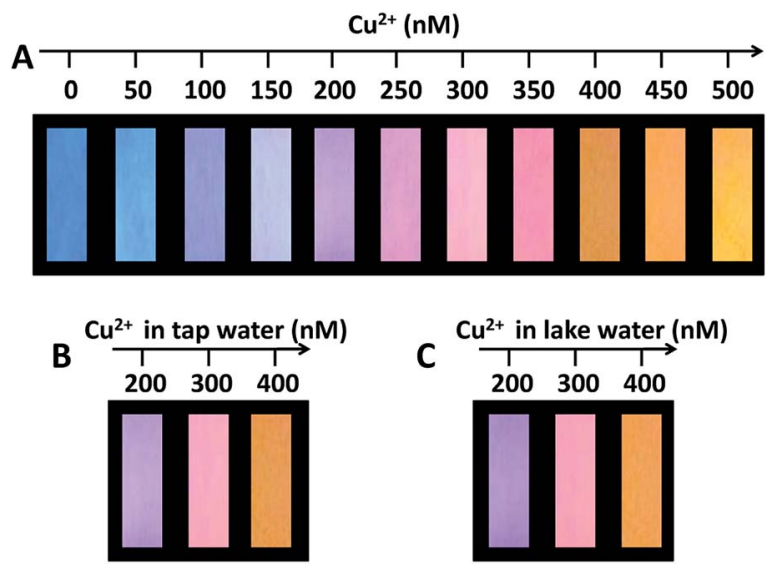

Fig. 4 (A) The visualization detection of $\mathrm{Cu}\left({ }^{\prime \prime}\right)$ using the fluorescent test papers prepared by printing dual colored CDs ink onto a piece of filter paper. (B and $\mathrm{C})$ The visual detections of $\mathrm{Cu}(\mathrm{II})$ in tap water and lake water, respectively. The photos were taken under a $365 \mathrm{~nm}$ UV lamp.

\section{Conclusion}

In summary, we have designed a dual-emissive fluorescent probe prepared by mixing BCDs and OCDs with fluorescence intensity ratio of $4: 1$ and its as-prepared text paper for the visual detection of $\mathrm{Cu}(\mathrm{II})$. With the addition of $\mathrm{Cu}$ (II), the variation of fluorescence intensity ratios produce an obvious change of the fluorescence color from blue to orange, which can be conveniently observed by the naked eye under a UV lamp without any complicated instrumentation. The detection limit of this probe reaches as low as $7.31 \mathrm{nM}$, which is much lower than the maximum level of $\mathrm{Cu}(\mathrm{II})(20 \mu \mathrm{M})$ in drinking water (EPA). The probe exhibits enhanced sensitivity and reliability of visual detection compared with single CDs-based probes, and possess many outstanding advantages, such as low toxicity, biocompatibility, low cost. Furthermore, we apply the ratiometric probe for visual identification of $\mathrm{Cu}(\mathrm{II})$ in real water samples. The results reported here prospect the bright future of fluorescent test paper for the feasible and inexpensive applications in chemical and biological sensing.

\section{Conflicts of interest}

There are no conflicts to declare.

\section{Acknowledgements}

This work is supported by National Natural Science Foundation of China (No. 21671052, 21371174), National Basic Research Program of China (2015CB932002), China-Singapore Joint Project (2015DFG92510) and Science and Technology Service Network Initiative of Chinese Academy of China (KFJ-SW-STS172).

\section{Notes and references}

1 Y. H. Zhang, H. S. Zhang, X. F. Guo and H. Wang, Microchem. J., 2008, 89, 142-147.

2 Z. Liu, S. Liu, P. Yin and Y. He, Anal. Chim. Acta, 2012, 745, 78-84.

3 Y. H. Chan, J. Chen, Q. Liu, S. E. Wark, D. H. Son and J. D. Batteas, Anal. Chem., 2010, 82, 3671-3678.

4 X. Sun, P. Liu, L. Wu and B. Liu, Analyst, 2015, 140, 67426747.

5 X. Liu, N. Zhang, T. Bing and D. Shangguan, Anal. Chem., 2014, 86, 2289-2296. 
6 C. Xie, L. Xiao, S. Peng and X. Shi, New J. Chem., 2014, 38, 6095-6102.

7 Y. Ding, S. Z. Shen, H. Sun, K. Sun and F. Liu, Sens. Actuators, $B, 2014,203,35-43$.

8 C. W. K. Chow, S. D. Kolev, D. E. Davey and D. E. Mulcahy, Anal. Chim. Acta, 1996, 330, 79-87.

9 J. Wu and E. A. Boyle, Anal. Chem., 1997, 69, 2464-2470.

10 M. S. Chan and S. D. Huang, Talanta, 2000, 51, 373-380.

11 Y. J. Zhou, X. Y. Huang, C. Liu, R. L. Zhang, X. M. Gu, G. J. Guan, C. L. Jiang, L. Y. Zhang, S. H. Du, B. H. Liu, M. Y. Han and Z. P. Zhong, Anal. Chem., 2016, 88, 6105-6109. 12 Y. H. Wang, C. Zhang, X. C. Chen, B. Yang, L. Yang, C. L. Jiang and Z. P. Zhong, Nanoscale, 2016, 8, 5977-5984.

13 X. Zhu, Z. Zhao, X. Chi and J. Gao, Analyst, 2013, 138, 32303237.

14 X. Zhao, J. Du, Y. Wu, H. Liu and X. Hao, J. Mater. Chem. A, 2013, 1, 11748-11753.

15 L. Cao, X. Wang, M. J. Meziani, F. S. Lu, H. F. Wang, P. G. Luo, Y. Lin, B. A. Harruff, L. M. Veca, D. Murray, S.-Y. Xie and Y.-P. Sun, J. Am. Chem. Soc., 2007, 129, 11318-11319.

16 D. R. Larson, W. R. Zipfel, R. M. Williams, S. W. Clark, M. P. Bruchez, F. W. Wise and W. W. Webb, Science, 2003, 300, 1434-1436.
17 R. L. Liu, D. Q. Wu, S. H. Liu, K. Koynov, W. Knoll and Q. Li, Angew. Chem., Int. Ed., 2009, 48, 4598-4601.

18 S. Y. Lim, W. Shen and Z. Gao, Chem. Soc. Rev., 2015, 44, 362381.

19 J. Wang, R. S. Li, H. Z. Zhang, N. Wang, Z. Z. Zhang and C. Z. Cheng, Biosens. Bioelectron., 2017, 97, 157-163.

20 K. Jiang, S. Sun, L. Zhang, L. Yue, A. Wu, C. Z. CaI and H. W. Lin, Angew. Chem., Int. Ed., 2015, 54, 5360-5363.

21 Y. H. Yuan, R. S. Li, Q. Wang, Z. L. Wu, J. Wang, H. Liu and C. Z. Huang, Nanoscale, 2015, 7, 16841-16847.

22 Y. H. Yuan, Z. X. Liu, R. S. Li, H. Y. Zou, M. Lin, H. Liu and C. Z. Huang, Nanoscale, 2016, 8, 6770-6776.

23 C. Liu, P. Zhang, X. Y. Zhai, F. Tian, W. C. Li, J. H. Yang, Y. Liu, H. B. Wang, W. Wang and W. G. Liu, Biomaterials, 2012, 33, 3604-3613.

24 H. Ding, S. B. Yu, J. S. Wei and H. M. Xiong, ACS Nano, 2015, 10, 484-491.

25 S. Ramaprabhu, J. Mater. Chem., 2012, 22, 18775-18783.

26 Y. L. Min, T. Wang, Y. G. Zhang and Y. C. Chen, J. Mater. Chem., 2011, 21, 6683-6689.

27 Y. Dong, R. Wang, G. Li, C. Chen, Y. Chi and G. Chen, Anal. Chem., 2012, 84, 6220-6224.

28 J. Yao, K. Zhang, H. Zhu, F. Ma, M. Sun, H. Yu, J. Sun and S. Wang, Anal. Chem., 2013, 85, 6461-6468. 